\title{
GW23-e1894 INITIAL AND LONG-TERM CLINICAL OUTCOMES OF UNPROTECTED LEFT MAIN STENTING USING DRUG ELUTING STENTS
}

doi:10.1136/heartjnl-2012-302920l.20

Yanhong Fan, Wenyi Guo, Weijie Li, Ronghua Luan, Anlin Lv, Ling Tao, Feng Cao, Yan Li, Chengxiang Li, Dianxin Zhang, Bing Liu, Oiong Wang, Hexiang Cheng, Yanjie Cao, Huan Li, Tao Hu, Fei Li, Zheng He, Haichang Wang. Department of Cardiology, Xijing Hospital, The Fourth Military Medical University

Objectives To evaluate the initial and long-term clinical outcomes of patients with unprotected left main (ULM) stenosis undergoing percutaneous coronary intervention (PCI) with drug eluting stents (DES) at XiJing Hospital Centre in real world patient population.

Methods After excluding acute ST-segment elevation myocardial infarction and bailout stenting, 798 patients treated for ULM disease with DES from January 2003 to December 2011 at XiJing Hospital were enrolled. The clinical outcomes of ULM treated with DES were evaluated by major adverse cardiac events (MACE) and stent thrombosis (ST) during in-hospital period and after long-term follow-up respectively. MACE was defined as cardiac death, non-fatal myocardial infarction (MI) and clinically driven target lesion revascularisation (TLR). ST was evaluated in accordance with the Academic Research Consortium (ARC) definitions.

Results The mean age of study population was $62 \pm 10$ years, 649 (81\%) patients were male, 199 (25\%) patients were diabetic, and the mean ejection fraction was $54 \% \pm 11 \% .71$ (9\%) patients had an ostial and 27 (3\%) had a shaft lesion (nondistal subgroup), 700 (88\%) patients had a bifurcation lesion (distal subgroup). In distal subgroup, $416(59 \%)$ patients were treated with 1 stent (1-stent subgroup) and $284(41 \%)$ were treated with 2 stent (2-stent subgroup). Among 2-stent subgroup, 74 (11\%) patients were treated with crush (crush subgroup), $86(12 \%)$ were treated with culotte (culotte subgroup), 43 (6\%) were treated with kissing (kissing subgroup) and 81 (12\%) were treated with $\mathrm{T}$ stenting ( $\mathrm{T}$ subgroup). Angiographic and clinical successes of PCI were obtained in all patients. During the inhospital period, MACE occurred in $7(0.8 \%)$ patients including 5 $(0.6 \%)$ cardiac deaths and $2(0.2 \%)$ MIs. one patient had definite (died) and four patients had probable stent thrombosis (1 had a $\mathrm{MI}$ and 3 died). During the long-term follow-up duration of 27 \pm 20 months, MACE occurred in 176 (22\%) patients including 25 (3\%) cardiac deaths, 16 (2\%) MIs and 135 (17\%) TLRs. six patients had probable (died) and 12 patients had possible stent thrombosis (2 had MI and 10 died). There were no significant differences in in-hospital MACE rates between nondistal and distal subgroup, between 1-stent and 2-stent subgroup and among different 2-stent technique subgroups. After long-term follow-up, the TLR rate was significantly higher in distal 


\section{ABSTRACTS}

subgroup than in nondistal subgroup (18\% vs $9 \%, p<0.05)$, the MACE rate was significantly higher in 2-stent subgroup than in 1 -stent subgroup $(28 \%$ vs $20 \%, p<0.05)$, and the different 2 -stent technique subgroups showed similar long-term outcomes. The total stent thrombosis rates were also no significant differences between nondistal and distal subgroup, between 1 -stent and 2 -stent subgroup and among different 2 -stent technique subgroups.

Conclusions Treatment of ULM with DES is feasible and safe with good in-hospital and acceptable long-term clinical results. Compared with ostial and shaft lesion, distal bifurcation lesion is associated with a worse long-term TLR rate. 1-stent technique had a better long-term MACE rate than 2-stent technique when distal bifurcation lesion was treated, whereas different 2 -stent techniques had similar clinical outcomes. 